\title{
In-plate metabolomics of single zebrafish embryos
}

\author{
Anton Ribbenstedt, ${ }^{1 *}$ Malte Posselt, ${ }^{1}$ Carl Brunius, ${ }^{2}$ and Jonathan P. Benskin ${ }^{1 *}$ \\ ${ }^{1}$ Department of Environmental Science and Analytical Chemistry (ACES), Stockholm \\ University, Sweden \\ ${ }^{2}$ Department of Biology and Biological Engineering, Chalmers University of Technology, \\ Sweden
}

*Corresponding authors:

Anton.Ribbenstedt@aces.su.se

Jon.Benskin@aces.su.se

Key words:

Non-target, REACH, OECD 236, Orbitrap, single embryo, 3R, metabolomics, MoA, mode of action, zebrafish embryo, propranolol, metaFET

\begin{abstract}
The fish embryo toxicity (FET) assay offers high-throughput and ethically favorable toxicity testing but does not yet meet the demands of REACH legislation. This is partly because the standard protocol for FET is largely incapable of generating data on a chemical's mode-ofaction (MoA). To address this limitation, we developed a seamless add-on to the FET assay involving high-throughput, single zebrafish embryo metabolomics ('metaFET'). Incubation, microscopy, embryo extraction, and instrumental metabolomics analysis of single zebrafish embryos were all performed in the same 96-well plate, following acquisition of conventional FET endpoints. The total time for metaFET (including testing of 6 doses with positive and negative controls, assessing conventional endpoints, instrumental analysis, data processing and multivariate statistics) is <14 days, i.e. 1 wk longer than conventional FET. Metabolomic perturbations at low dose were linked statistically to those observed at high dose and in the presence of an adverse effect, thereby contextualizing omic data amongst regulatory endpoints.
\end{abstract} Overall, this technology enables collection of high resolution metabolomic data in a high 
throughput manner, suitable for MoA hypothesis generation in the context of regulatory pharmaceutical or toxicological screening.

\section{Introduction}

EU REACH (Registration, Evaluation and Authorization of Chemicals) legislation requires chemical manufacturers to carry out comprehensive hazard assessment on new products, necessitating the use of large numbers of animals (11.5 million in 2011) in the European Union alone $^{1}$. In addition to the practical challenges associated with high throughput toxicity testing (e.g. time, money) there are ethical dilemmas associated with experimentation involving animals. Recently, the United States Environmental Protection Agency pledged to stop financing animal tests by $2035^{2}$ and within the EU REACH legislation lies imbedded the $3 \mathrm{R}$ principle which strongly encourages a reduction in animal tests ${ }^{3}$. Substitution of the OECD adult fish acute toxicity test (test guideline (TG) 203) with the fish embryo acute toxicity test (TG 236) could address some of these issues ${ }^{4}$ through low cost, multi-well plate-based in vitro testing ${ }^{5}$. However, TG 236 does not yet meet the information requirements of REACH Annex VIII on its own, in part because apical endpoints measured by TG236 are not fully compatible with those of TG203 ${ }^{6}$. Thus, TG236 is currently only suitable for regulatory purposes by applying a weight-of-evidence approach. For example, if a chemical's mechanism-of-action (MoA) is known, inclusion of TG236 data may fulfill the requirements of REACH for shortterm acute toxicity in fish. MoA data are necessary for linking molecular initiating events to apical endpoints through Adverse Outcome Pathways and a number of studies have generated chemical-specific and mixture-exposure transcriptomic ${ }^{7-10}$, proteomic ${ }^{11,12}$, and metabolomic data $^{10,13}$ from zebrafish embryos. However, these data are often of limited use to regulators, since incubation protocols are usually non-standardized (and do not follow TG236), and omic 
responses are often not connected to an apical endpoint, making them difficult to interpret in a regulatory context. ${ }^{14}$

Most metabolomic studies on zebrafish (ZF) embryos have employed pooling approaches, extracting between 3 and 80 embryos per replicate, in order to obtain sufficient biomass for instrumental analysis ${ }^{10,13,15-17}$. One of the major drawbacks of pooling is that there is no practical way of linking apical endpoints of individual embryos to their respective exposureinduced metabolic perturbations. While the analysis of single ZF embryos could facilitate direct linkages between metabolic perturbation and apical endpoints, single ZF embryo metabolomics is hampered by their small size ( $200 \mu \mathrm{g}$ /individual at $120 \mathrm{hpf})$, which makes them difficult to extract and ultimately generate sufficient quantities of extract for instrumental analysis. Perhaps for this reason, single ZF embryo metabolomics has, to our knowledge, only been attempted once, with a focus on studying changes in the metabolome of developing embryos ${ }^{18}$ (i.e. not conducted in resemblance to TG236). In that work, a 3-step homogenization scheme was followed by filtration and pre-concentration prior to LC- and GC-MS analysis ${ }^{18}$. However, preparing samples in this manner is time consuming, and limits the number of replicates which are practically possible to analyze.

Here we present an addition to the FET assay which rapidly generates and contextualizes metabolomic data amongst conventional toxicological endpoints. Incubation, microscopy, embryo extraction, and instrumental analysis were all performed in the same 96-well plate, resulting in a total assay time of less than 2 weeks ( 1 week longer than the standard 236 assay). Metabolomic effects at low dose are statistically linked to those at high dose (i.e. in the presence of an apical endpoint) which places these data into context amongst regulatory endpoints. Overall, this sample preparation and analysis method is suitable for high sensitivity, high 
throughput MoA hypothesis generation in regulatory pharmaceutical or toxicological screening.

\section{Materials and methods}

\section{In-plate heart rate, morphology, and mortality measurements according to TG 236}

ZF embryos were incubated up to 120 hours post fertilization (hpf) with the beta blocker propranolol (PPL) building on the OECD 236 Test Guidelines ${ }^{4}$. A single plate consisted of 6 different concentrations of PPL ( $\mathrm{n}=12$ embryos/concentration), a vehicle control $(\mathrm{n}=12$ embryos) and a positive control (3,4-dicholoroaniline (DCA); n=12 embryos; see Figure S1 for plate layout). At 48 and 120 hpf we examined embryos microscopically for standard lethal and sub-lethal apical endpoints (plus heart rate ${ }^{19,20}$; Table S1) and observed significantly lower heart rates (relative to negative controls) in both the positive control and the $46540 \mu \mathrm{g} / \mathrm{L}$ exposure group at $48 \mathrm{hpf}$ (Fig. 1a), as well as the 46540 and $4550 \mu \mathrm{g} / \mathrm{L}$ groups at $120 \mathrm{hpf}$ (Fig. 1b). These results align well with the only no- and lowest-observed effect concentrations (NOEC and LOEC, respectively) for decreased heart rate due to PPL exposure in zebrafish embryos $\left(3500 \mu \mathrm{g} / \mathrm{L}\right.$ and $7000 \mu \mathrm{g} / \mathrm{L}$ respectively at $\left.48 \mathrm{hpf}^{20}\right)$ known to the authors. Significant mortality was only observed in the positive controls, both at 48 and $120 \mathrm{hpf}$.

\section{Dosage analysis}

By measuring the exposure water in each well, following incubation and exposure, we were able to determine the concentrations for all but the lowest dose (which was below the limit of quantification; Table S2). The measured concentrations in the exposure medium were on average $0.49,12,62,4550$ and $46540 \mu \mathrm{g} / \mathrm{L}$ at $120 \mathrm{hpf}$. The nominal dose for the lowest concentration was $0.050 \mu \mathrm{g} / \mathrm{L}$. We also confirmed that doses stayed within $\pm 10 \%$ over the 
duration of exposure, thus ruling out substantial degradation or biotransformation during the study (Table S3).

\section{In-plate sample preparation and sequence planning}

To minimize sample handling and enhance throughput for metabolomic analysis, we developed a method for in-plate extraction of up to several hundred embryos in $<3$ hours. The extraction involved bead blending and sonication with methanol/chloroform (plus internal standard) followed by centrifugation (see Supporting Information), after which the plate was placed directly in the autosampler of the liquid chromatograph (LC) for instrumental analysis. We used mixed-diameter stainless-steel beads to reproducibly homogenize the embryos, as opposed to single-sized beads which tended to leave the larvae husk intact. By gluing silicone cap-mats lined with polytetrafluoroethylene (PTFE) to each 96-well plate, up to 960 embryos (i.e. $10 \times 96$ well plates stacked) could potentially be homogenized simultaneously with this method. To ensure the absence of cross contamination between wells, we performed a number of a priori microscopy experiments where no sign of moisture between the wells was detectable and where empty wells close to solvent-filled wells remained dry after homogenization. Blanks were prepared in separate blank plates alongside the sample plates, but followed the exact same treatment as normal samples, and were injected prior to the analysis of real samples.

In order to avoid compromising the LC columns and mass spectrometer through injection of small, insoluble skin-fragments we increased the autosampler needle-height to draw extract 5 $\mathrm{mm}$ from the bottom of the wells (as opposed to the default setting $2 \mathrm{~mm}$ ). In this step, the extraction beads also served to stop suction-induced turbulence from disturbing tissue remnants at the bottom of the wells. As a final precaution, we installed an in-line metal filter $(0.5 \mu \mathrm{m})$ downstream of the autosampler but upstream of the analytical column. Each plate underwent 
three analyses over 74 hours: a) a lipidomics analysis via flow-injection MS/MS, b) non-target analysis using hydrophilic interaction chromatography (HILIC) positive ionization Orbitrap mass spectrometry; and c) resuspension followed by reversed phase-chromatography negative ionization Orbitrap mass spectrometry (see Supporting Information).

Signal-drift during both lipidomic and Orbitrap analyses were corrected using the R-package batchCorr $^{21}$ which required inclusion of quality control (QC) samples throughout the injection sequences. The QCs were prepared in $1.5 \mathrm{~mL}$ polypropylene tubes and consisted of extract of five pooled embryos, for which the same solvent to sample ratio as for plate samples was used. Blanks for the QCs were prepared in $1.5 \mathrm{~mL}$ polypropylene tubes and analyzed together with the plate blanks. Furthermore, we analyzed a portion of the highest concentration dose medium with both Orbitrap methods to identify features corresponding to PPL (e.g. parent ions, insource fragments, and impurities), and remove them during processing. Collectively, analysis using all three methods yielded over 13,000 raw features per embryo.

\section{Isolation of endogenous metabolites through data filtration}

Both lipidomic and Orbitrap data were processed using previously described methods developed by our group ${ }^{22}$, the former of which was adapted from Liebisch et $\mathrm{al}^{22,23}$ (see also Supporting Information). Peaks acquired through lipidomics analysis had to be on average 10fold higher than in blanks to be considered metabolite features. For non-target data, exogenous substances (e.g. PPL, its metabolites and background noise) were removed using a combination of batchCorr ${ }^{21}$ and an in-house $\mathrm{R}$ package comprised of a total of 6 data filters ${ }^{24}$. The first filter used the gap-filling status recorded by Compound Discoverer (CD) to remove features not detected in any of the sequence QC injections. Features which passed the gap-filter were then subjected to sequence correction using batch $\operatorname{Corr}^{21}$ and any features displaying RSDs above 
$30 \%$ in the sequence QCs post correction were discarded. The third filter removed low-intensity features (i.e. those features with highest peak height $<200,000$ counts per second for ESI+ HILIC or $<150,000$ counts per second for ESI- reversed-phase) whereafter the remaining features were internal standard-corrected to account for inter-well variability in evaporation. For the fourth filter we used the annotation package "ramclustR" to detect and remove any insource fragments of the masses predicted to be metabolites of PPL by CD, based on retention time and MS1 intensities ${ }^{25}$. The fifth filter removed all features within $5 \mathrm{ppm}$ of masses on a list of expected phase I and II metabolites of PPL generated by CD, as well as all features detected in the exposure medium and in blanks. The sixth filter removed any feature which had negative intensities and the seventh filter discarded features present in procedural blanks with peaks $>40 \%$ relative to the maximum signal in samples or QCs respectively. Overall, this procedure reduced the total number of features obtained from lipidomic and Orbitrap analyses from $>13000$ to $<350$ (Table S4).

\section{Multivariate statistical analysis}

Statistical analysis for apical observations were carried out using Student's t-test for heart rates and Pearson's Chi-squared test for mortality. For metabolomics data statistical analysis was performed using the R-package $\mathrm{MUVR}^{26}$ which performs minimal variable selection through recursive variable elimination by repeated double cross-validation (Table S5). In order to both characterize the MoA of PPL and to obtain data suitable for benchmarking of exposure, we developed and refined two random forest (RF) models: a classification and a regression model.

\section{Metabolite identification through retention time and MS2-based fingerprint matching}

Metabolite features measured by non-target analysis and selected by the models were putatively identified using mzCloud (through CD), Metlin ${ }^{27}, \operatorname{Metfrag}^{28}$ and 
Sirius+CSI:FingerID ${ }^{29,30}$. For compounds where there was no clear consensus regarding identification between these four software, we used the structure proposed by Sirius+CSI:FingerID due to its performance in inter-method comparisons ${ }^{31}$. Following putative identification, we procured authentic standards and determined a similarity score between sample and standard MS2 spectra using the R-script 'NTScreeneR' ${ }^{32}$ (Data S1). All candidate metabolite features displayed a similarity score $>0.85$, which we deemed sufficient to confirm their identities.

\section{Results and Discussion}

\section{Reproducibility of between-plate controls}

To evaluate the potential for future cross-plate comparison, and the reproducibility of our inplate sample preparation method, we analyzed non-exposed (i.e. control) embryos distributed over four plates from a second incubation event (see Methods). After instrumental analysis (i.e. Orbitrap using HILIC chromatography and lipidomics) and a data processing approach slightly modified to facilitate batch comparison (see Methods) we used principle component analysis (PCA) to get an unbiased measure of between-plate variability in the measured metabolome. Evaluating the PCA score plots for both the lipidomics (Fig. 2a) and Orbitrap data (Fig. 2b), as well as a combination thereof (Fig. 2c) it is not surprising that only the Orbitrap data scores plot shows signs of outliers (Fig. 2a). Non-target metabolomics is not only susceptible to insequence drift but also drift between batches, both in mass and baseline signal ${ }^{21}$. To counteract these phenomena we prepared a batch QC sample which, together with batchCorr, was used to normalize the baseline between the Orbitrap data of the four plates ${ }^{21}$. Since the outliers are not present in the lipidomics data (Fig. 2b) we conclude that the deviation of the outliers is not inherent to the embryos or the sample preparation but rather stems from the analysis. Interestingly, the extreme samples in the Orbitrap data are almost completely attenuated in the 
first 2 PCs by combining the datasets (Fig. 2c). Altogether, by utilizing the full functionality of batchCorr $^{21}$, we were able to show that zebrafish embryos can be reproducibly incubated, homogenized and analyzed using our in-plate sample preparation and analysis methods.

\section{PPL exposure modelling}

For the classification model we used all PPL exposure concentrations as well as negative controls as input which generated a model based on 34 of the 311 metabolite features. Unsurprisingly, the majority of misclassification of samples occurred between similar dosing concentrations (i.e. doses within 1 order of magnitude; Fig. 3a). However, when we considered misclassification as only those samples which were incorrectly classified by over an order of magnitude, the misclassification-rate decreased from $51 \%$ to only $24 \%$ (Fig. 3a). Permutation analysis of the model with randomized sample labels revealed that the model was highly statistically significant $\left(n=100, p=1.16 \times 10^{-10}\right)$ which confirmed that there was no overfitting. In comparison, the RF regression model was constructed using only the negative control along with the groups in which statistically significant apical endpoints were observed (i.e. heart rate perturbations at 46540 and $4550 \mu \mathrm{g} / \mathrm{L}$; Fig. 3b). This model resulted in a selection of 10 metabolite features, 7 of which were also identified by the classification model (Fig. 4). The Q2 and p-value for 100 permutations were 0.67 and $9.2 \times 10^{-5}$ respectively. In summary, we obtained two adequately accurate and non-overfitted RF models of PPL exposure using MSdata collected from single ZF embryos through RF analysis.

\section{PPL mode of action}

The relative, scaled abundance of the 11 structurally confirmed, 20 putatively identified, and the 6 unidentified metabolite features elected by the two models were plotted on a heat map to obtain a biochemical overview of the exposure (Fig. 4). Both monotonic and non-monotonic 
dose-responses were observed, highlighting the multi-faceted and complex perturbation of the metabolome caused by PPL exposure. It is well-documented that PPL strongly interacts with the phosphatidate phosphatase (PAP)/phospholipase D (PLD) pathway, causing inhibition of PAP and induction of PLD (Fig. 5a) ${ }^{33-35}$. The PAP/PLD pathway is a major component of glycerophospholipid metabolism in which a number of other enzymes have been documented to be affected by PPL exposure (Fig. 5a). Phospholipids such as phosphatidylcholine (PC), ethanolamine (PE), -inositol (PI), -serine (PS), -glycerol (PG) and phosphatidic acid (PA) as well as the fatty acids (FA) to which they are composed of are not only structural components of the lipid membrane but also signaling molecules governing important cellular and physiological mechanisms such as cytokinesis ${ }^{36}$, apoptosis $^{37}$, neurotransmission ${ }^{38}$ and inflammation. ${ }^{39}$ Thus, it is no surprise that a large number of PC lipids show signs of a monotonic increase in abundance over the exposure doses in this study (Fig. 4). Interesting to note is that our RF regression model is solely based on compounds with an increase in relative concentration over increasing exposure doses. We hypothesize that the chain of events that induces PC lipids with one ether-bound FA also explains the increase of PE lipids in higher exposure doses (Fig. 4). When PPL induces PLD and inhibits PAP a build-up of PA will occur ${ }^{35}$ which will lead to a subsequent increase in PI and PS ${ }^{40}$. PS can be further metabolized into PE which in turn can be metabolized into glycerol-3-phosphate, one of the main starting molecules for ether lipid metabolism (Fig. 5a). Also, many enzymes which are either directly or indirectly affected by PPL can be found within the ether lipid metabolism pathway (e.g. phospholipase A2 (PLA2) ${ }^{41}$, PAP, PLD and PLC ${ }^{42}$ ). So, despite the lack of detailed knowledge on how PPL interacts with ether lipid metabolism, our findings in literature are indicative of a MoA which would explain the stepwise increase and subsequent decline in PE and the monotonic increase in PC ether lipids over the exposure concentrations. A number of biologically important FAs (i.e. docosapetaenoic acid, eicosapentaenoic acid, docosahexaenoic acid, X-hydroxy 
eicosatetraenoic acid) showed similar non-monotonic expression patterns to one another, consistent with the response observed for a number of lysoPEs and lysoPCs (Fig. 4). The concentration of these FAs decreased in the lower dose groups $(0.5-62 \mu \mathrm{g} / \mathrm{L})$ before returning to levels similar to controls at the highest doses $(4540-46540 \mu \mathrm{g} / \mathrm{L})$. This could be explained by the ability of PPL to inhibit the activation of PLA2, which facilitates hydrolysis of acylbound FAs from phospholipids (Fig. 5a) ${ }^{41}$. Considering that all of the affected lipids and FAs are messenger molecules, we propose that fold-change increases in higher doses might be the result of many negative and positive feedback loops interacting with each other. The only nonlipid related metabolite feature we identified with a level 1 certainty was phenylalanine, which has also been reported in a previous metabolomics study on PPL exposure ${ }^{43}$. The direct inhibition of tyrosine hydroxylase (TH) by PPL is a likely explanation for the relative increase of phenylalanine in the two highest exposure concentrations in our data (Fig. 5b) ${ }^{44}$. If tyrosine concentrations grow large, due to TH-inhibition, alternative pathways could be over-burdened which would ultimately result in a build-up of its precursor phenylalanine. Thus, we conclude that the metabolites elected by our models are consistent with existing literature on PPL and provide strong evidence supporting their perturbation at the exposure concentrations investigated in the present work.

\section{Ecotoxicological evaluation}

Having confirmed that our models reflected actual metabolomic perturbations we employed the RF regression model to predict the four concentrations not used to train the model (i.e. 0.05 $\mu \mathrm{g} / \mathrm{L}, 0.49 \mu \mathrm{g} / \mathrm{L}, 12 \mu \mathrm{g} / \mathrm{L}$ and $62 \mu \mathrm{g} / \mathrm{L}$; Fig. 6). For the test-set dose-groups we then calculated the Q2-value for all exposure groups together and separately (Equation S1). The results showed a large variation in predictability of different dose concentrations (Fig. 6): The two higher doses $(12 \mu \mathrm{g} / \mathrm{L}$ and $62 \mu \mathrm{g} / \mathrm{L})$ were very close to modelled values while lower doses $(0.05 \mu \mathrm{g} / \mathrm{L}$ and 
$0.49 \mu \mathrm{g} / \mathrm{L}$; Fig. 5) suffered from slightly worse prediction accuracy, although without any measurable bias (Fig. 6). Importantly, predicted doses at all concentrations were significantly higher than predictions of the negative control, suggesting a high potential to correctly identify the occurrence of exposure even at such low exposures that could not be accurately predicted (Fig. 6). We also used the data acquired from the model to make benchmark dosing (BMD) calculations using the U.S. EPA BMD tool (BMDS, version 3.0) $)^{45}$. This resulted in a lognormal model with constant variance which fulfilled all but one of the default requirements of BMDS (Table S6). The metabolomic BMDL and LOEC of this model were $0.0016 \mu \mathrm{g} / \mathrm{L}$ and $0.050 \mu \mathrm{g} / \mathrm{L}$ at $120 \mathrm{hpf}$, which are orders of magnitude lower than the LOEC for heart rate (7000 $\mu \mathrm{g} / \mathrm{L}$ at $48 \mathrm{hpf}^{20} ; 46540 \mu \mathrm{g} / \mathrm{L}$ at $48 \mathrm{hpf}$ in this study) which the metabolomic effect is anchored in. Unfortunately, we were unable to find any heart rate BMDL for propranolol in the literature.

\section{Perspectives on the use of metaFET for regulatory hazard assessment}

While the standard FET assay generates hazard data based on mortality (e.g. NOEL, LC50), it provides little in the way of mode-of-action. This limitation is addressed in the metaFET approach by linking metabolomics data to the standard FET assay, which represents a significant leap forward in chemical hazard assessment. Previous protocols for ZF embryo LCMS analysis have decoupled apical and metabolomic endpoints, by requiring the pooling of embryos. Our approach represents a vast improvement by generating apical and metabolomic measurements from the same embryo. The metaFET in-plate extraction and analysis adds on to standard FET assays without the need to perform additional embryo experiments. The extraction procedure, while minimizing sample handling error induced by multi-step protocols and the delicacy of small sample volumes, also improves time-efficiency, thus effectively enabling large-scale, high-throughput analysis. The possibility to determine metabolomic BMDs, NOECs and LOECs linked to apical endpoints in single fish embryos not only forwards 
the possibility to use omics data in a legislative context but it also emphasizes the question of where the threshold of an adverse effect should be drawn. Beyond molecular hazard screening, the developed in-plate sample processing method can also be used for rapid determination of transformation products, metabolomics of fish embryos from other fish species, other small test organisms or cells as well as processing of smaller samples from larger organisms which require small extraction volumes. Overall, the method presented here constitutes a significant development in high-throughput, cost-effective LC-MS analysis of small organisms.

\section{Acknowledgment}

We thank the SciLife lab facility in Uppsala, Sweden for providing their expertise, embryos and labs to perform the incubation and apical analysis.

\section{Author contributions}

Anton Ribbenstedt: Project conceptualization, experimental design, incubation and exposure of zebrafish embryos, sample preparation, instrumental analysis, statistical analysis, pathway perturbation analysis, interpretation of results, manuscript preparation.

Malte Posselt: Contributions to experimental design, incubation and exposure of zebrafish embryos, dose analysis, interpretation of results, manuscript preparation.

Carl Brunius: Statistical analysis, interpretation of results, manuscript preparation.

Jonathan P. Benskin: Project conceptualization, experimental design, statistical analysis, interpretation of results, manuscript preparation.

\section{Competing interests}

The authors declare no competing interests. 


\section{Data availability}

Datasets obtained through data processing of instrumental raw files from Thermo Scientific Quantiva and from Q Exactive Orbitrap instrumental analysis are available in the Data Dryad repository (https://doi.org/10.5061/dryad.7m0cfxppz). The raw files generated through instrumental analysis in this study, as well as result files generated by data processing software, are available from the corresponding author upon reasonable request.

\section{Code availability}

All code involved in formating data prior to employment of functions included in the "batchCorr" R-package are available through requests sent to the corresponding authors. Code related to reorganization and deconvolution of lipidomics data is available as a package for $\mathrm{R}$ called "metLab", (https://github.com/parasitetwin/metLab). All functionality connected to our non-targeted data filtering procedures are available as a package for R called "ExpMetFilter", (https://github.com/parasitetwin/ExpMetFilter). 

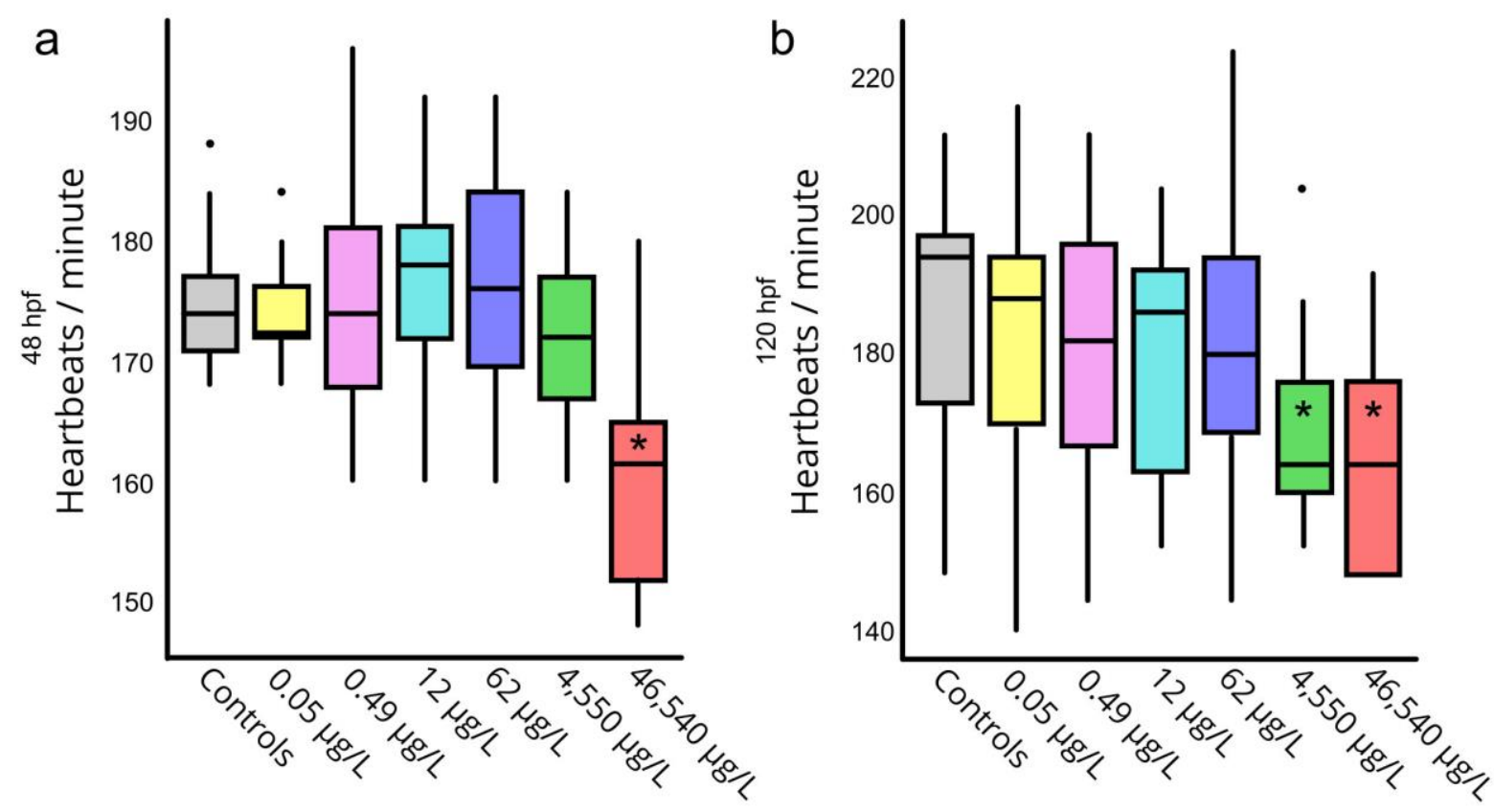

Fig. 1: Heart rate of embryos measured at $48 \mathrm{hpf}(\mathrm{A})$ and $120 \mathrm{hpf}(\mathrm{B})$ for the range of exposure concentrations. Heart rate was measured through microscopy and determined by counting the number of heartbeats of the embryo during 15 seconds. Groups with significantly lower heart rate compared to controls $(\mathrm{p}<0.05)$ are marked by $*$. 

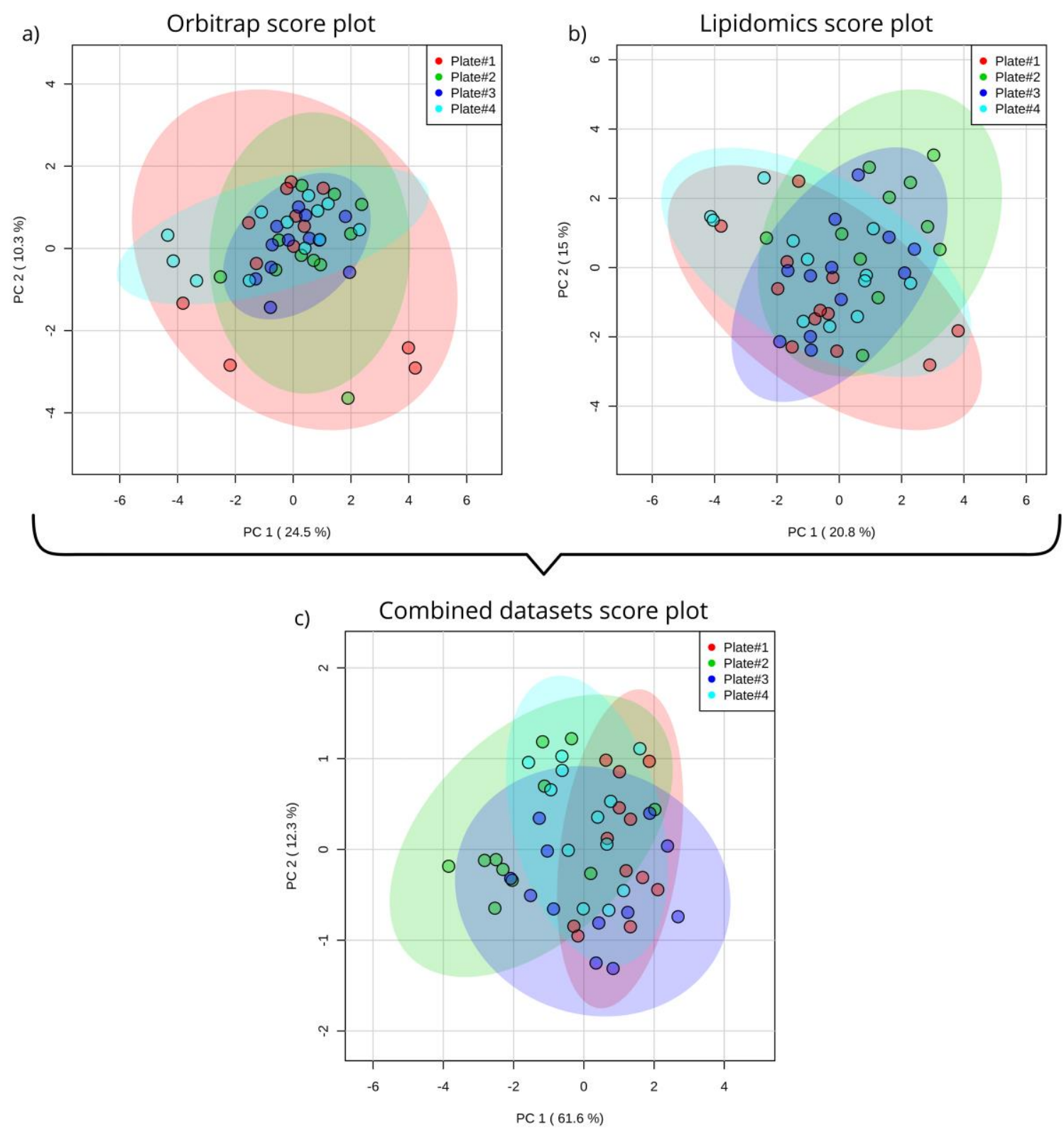

Fig. 2: PCA score plot of single zebrafish embryo data from four different plates acquisitioned by Orbitrap utilizing HILIC chromatography (A); lipidomics analysis (B) and a combination of both analyses (C). 
a

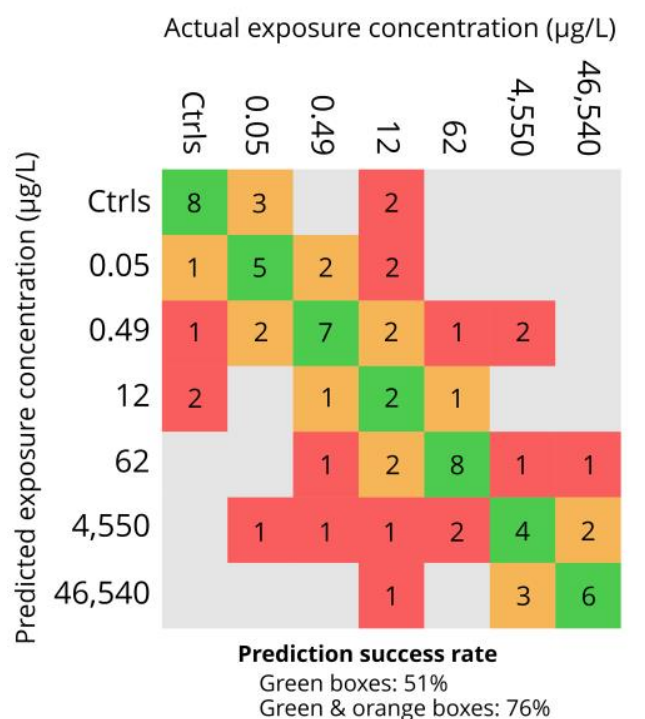

b

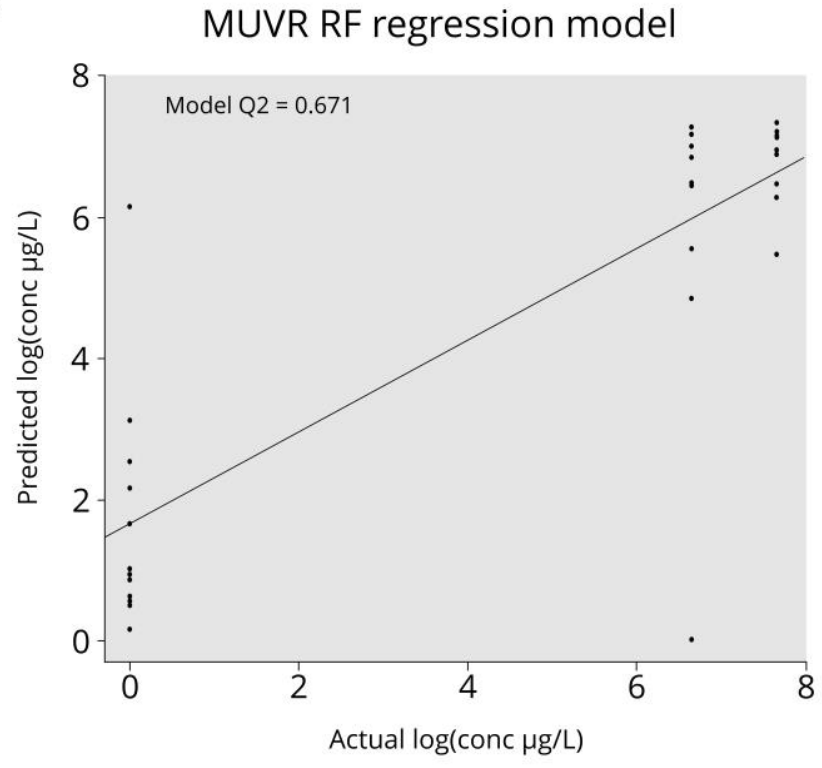

Fig. 3: Results of MUVR random forest (RF) modelling. Panel A shows results of the RF classification model with actual and predicted exposure groups in the columns and rows, respectively (green for correct predictions, orange for prediction within one order of magnitude and red for erroneous predictions). Panel B shows the results of the RF regression model, trained on the two exposure groups with lower heart rate at $120 \mathrm{hpf}$ (i.e. 4550 and $46540 \mu \mathrm{g} / \mathrm{L})$ and the negative controls $\left(\mathrm{Q} 2=0.67, \mathrm{p}=9.2 \times 10^{-5}\right)$. 


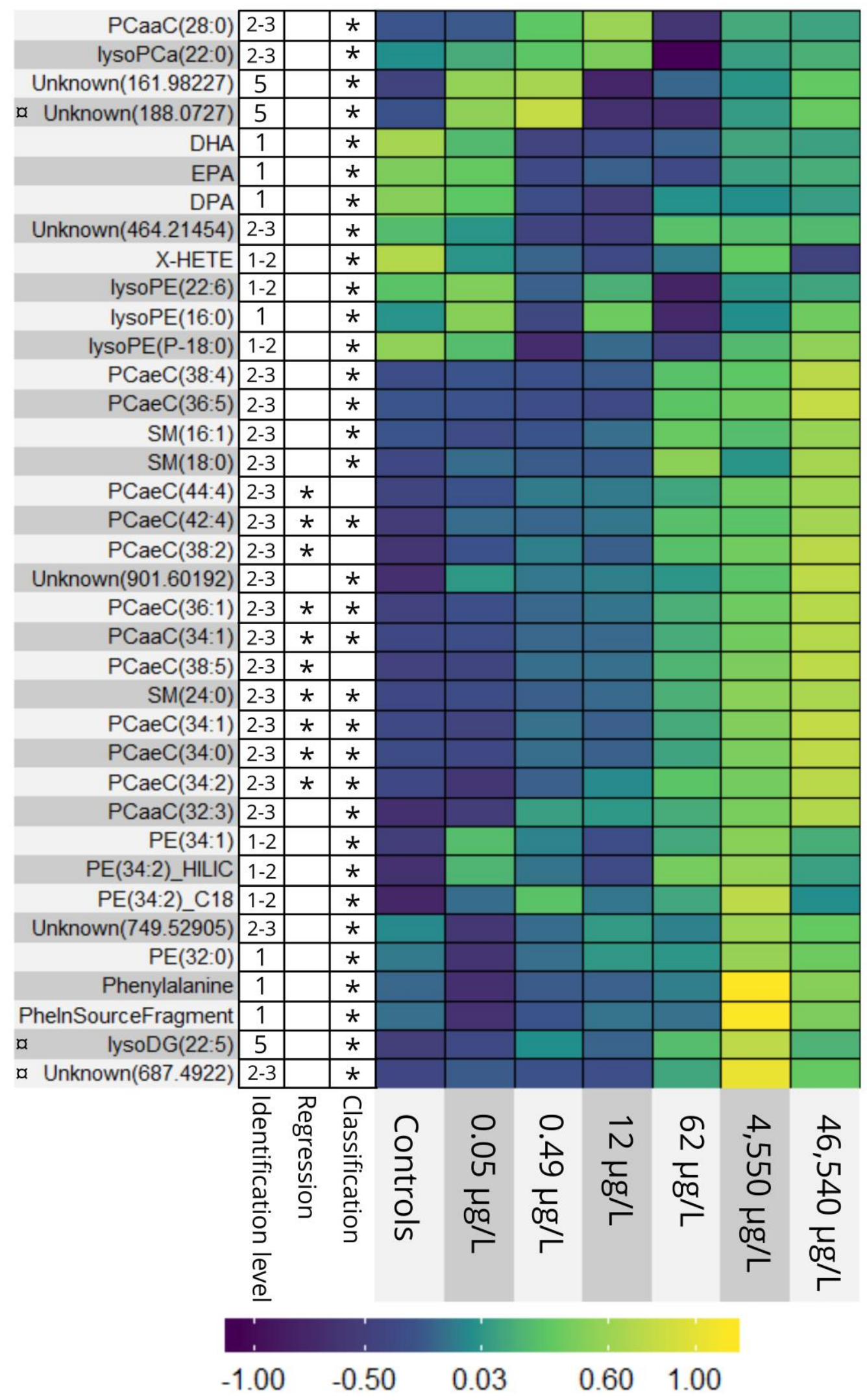

Fig. 4: Heatmap of all endogenous metabolites elected by the classification and regression random forest models. 1-5 = level of putative identification as suggested by Schymanski et al. $^{46} \mathrm{a}=$ No MS2 data gathered. 


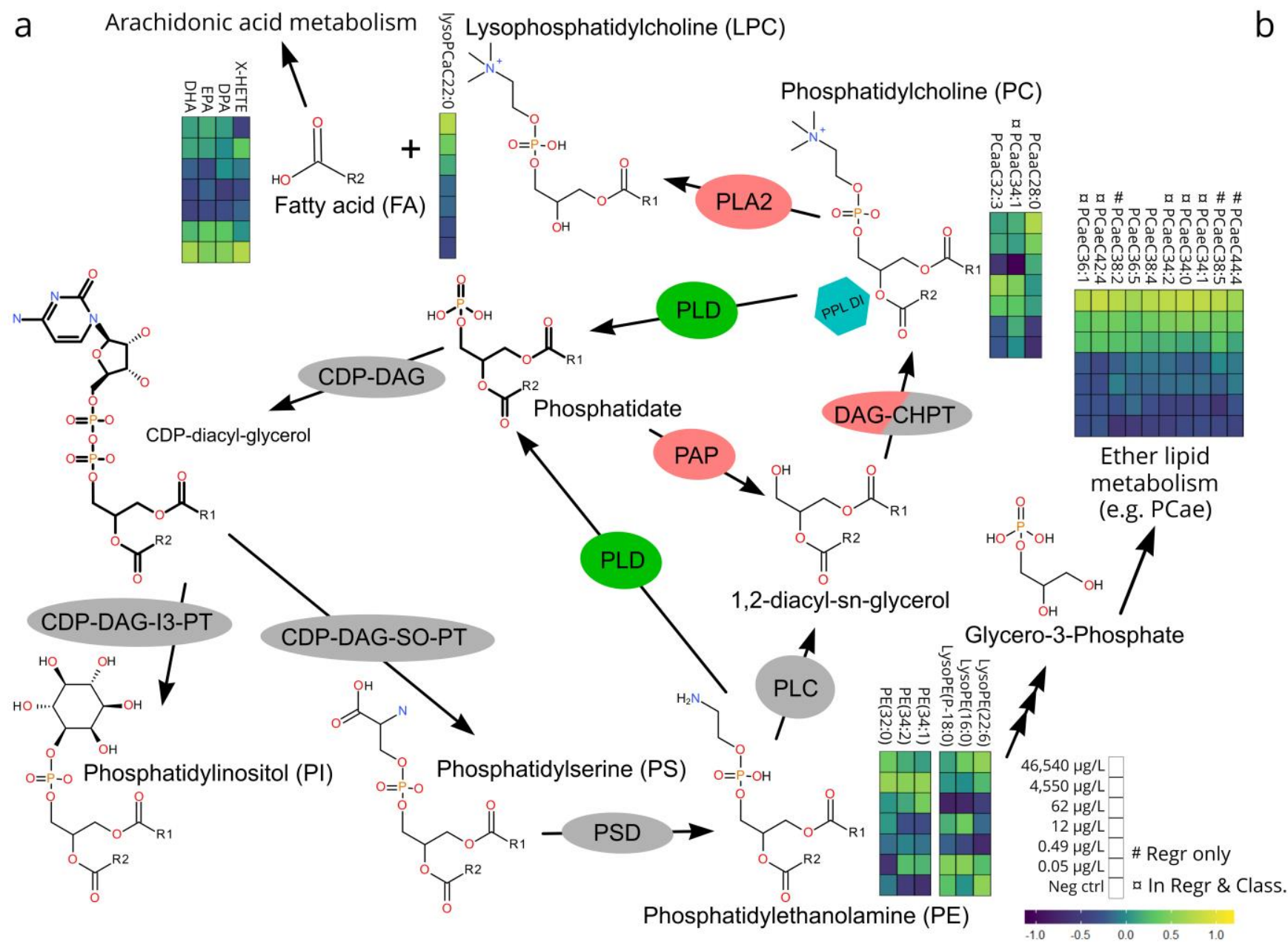

b
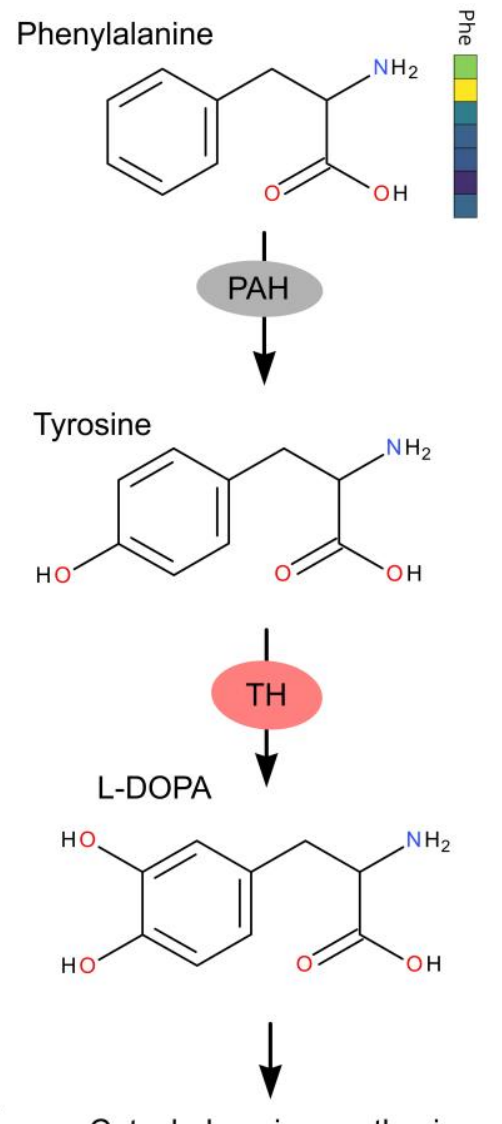

Catecholeamine synthesis

Fig. 5: Pathways of phospholipid (A) and tyrosine (B) metabolism implicated in the mode of action of propranolol. Enzymes are color-coded for up-regulation or induced activity (green), down-regulation or reduced activity (red) or no documented effect (gray) associated with PPL. The hexagon indicates a direct interaction of PPL (PPL DI) with phopshatidylo choline lipids. Metabolite heatmaps are arranged from the highest concentration (top) to the negative control (bottom). \# = Only in regression model; $\alpha=$ Both in regression and classification model. 


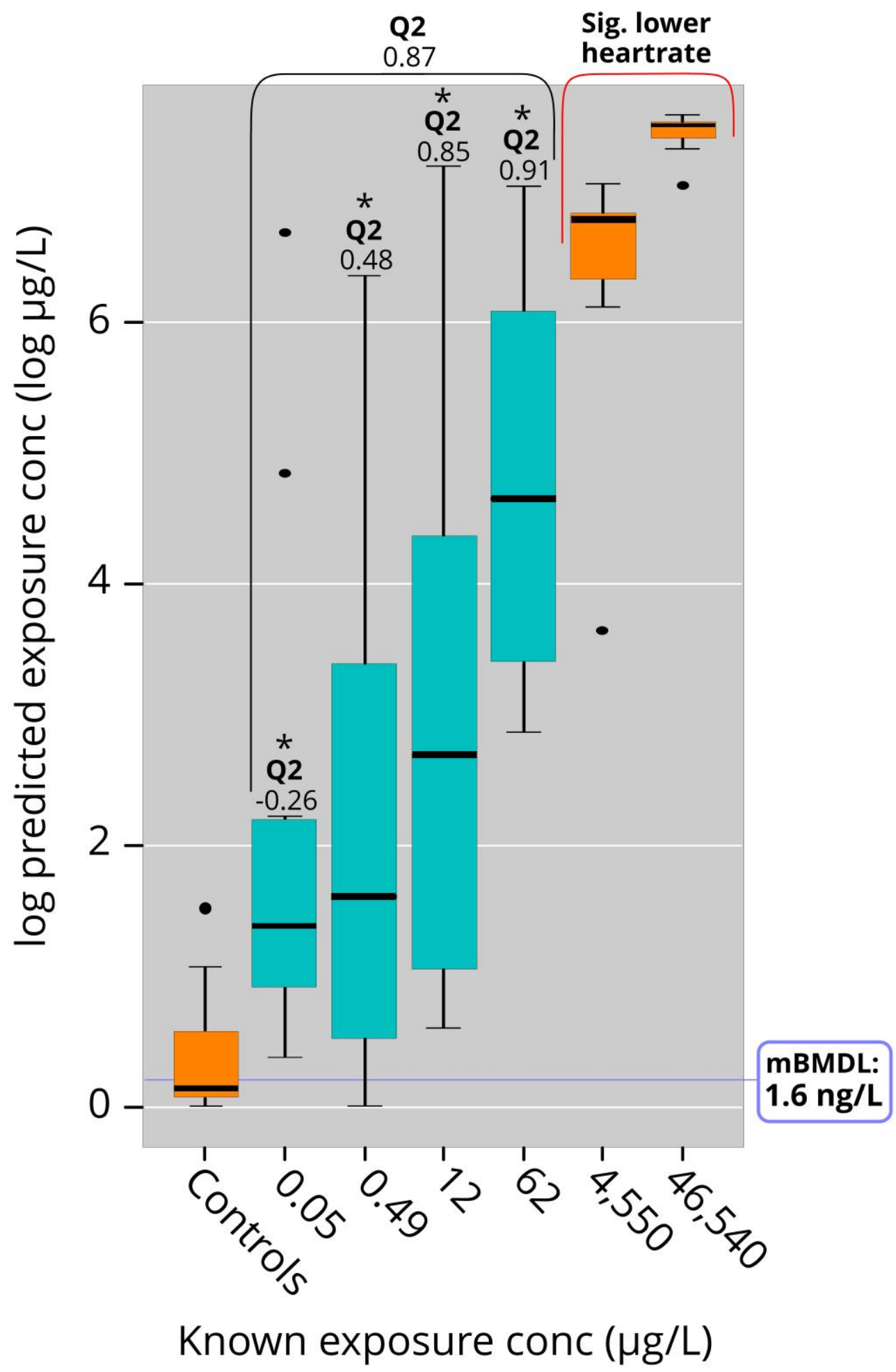

Fig. 6: Exposure concentration predicted by the MUVR RF regression model trained on exposure groups marked in orange. Exposure groups marked in turquoise were not used for model construction, but still produced dose-dependent predictions. Concentrations predicted to be significantly $(\mathrm{p}<0.05)$ lower than $4550 \mu \mathrm{g} / \mathrm{L}$ and higher than negative controls are denoted with *. Of note, although predictions at the lowest exposure $(0.05 \mu \mathrm{g} / \mathrm{L})$ were inaccurate, the model still managed to identify these samples as exposed. mBMDL is the lower metabolomics benchmarking dose as determined by the US EPA tool "BMDS 3.0" using prediction data from the regression model. 


\section{Supporting information}

Data S1 - All similarity scoring results from 'NTScreener'

Equation S1 - Equation used to calculate Q2 scores for regression model predictions

Figure S1 - Plate layout for the exposure in the 96 well plate

Notes S1 \& S2 - Full description of Compound Discoverer processing methods used for peak picking and integration of both datasets (HILIC \& C18 chromatography)

Table S1 - Raw data for all apical endpoints

Table S2 - Concentration of propranolol in the exposure plate

Table S3 - Measured concentrations of degradation plate at 0, 48 and $120 \mathrm{hpf}$

Table S4 - The number of features removed from the non-target datasets by each filter applied to the raw dataset

Table S5 - Meta-information on the setup of MUVR for generating the two random forest models

Table S6 - Result file from US EPA tool "BMDS 3.1" analysis using random forest regression model predictions of embryos

Table S7 - Water conditions in SciLife facility in Uppsala

Text S1 - Detailed methods containing everything needed to reproduce study 


\section{References}

(1) European Commission. "Report from the Commission to the Council and the European Parlament”, COM(2013) 859 Final, Available at Https://EurLex.Europa.Eu/Procedure/EN/1041492 (Accessed in May 2019).; COM(2013); 859.

(2) US EPA, O. Administrator Wheeler Signs Memo to Reduce Animal Testing, Awards \$4.25 Million to Advance Research on Alternative Methods to Animal Testing https://www.epa.gov/newsreleases/administrator-wheeler-signs-memo-reduce-animaltesting-awards-425-million-advance (accessed Sep 18, 2019).

(3) Čihák, R. REACH - an Overview. Interdiscip. Toxicol. 2009, 2 (2), 42-44. https://doi.org/10.2478/v10102-009-0007-1.

(4) OECD. Test No. 236: Fish Embryo Acute Toxicity (FET) Test; Organisation for Economic Co-operation and Development: Paris, 2013.

(5) White Paper, Reach.Pdf.

(6) Sobanska, M.; Scholz, S.; Nyman, A.-M.; Cesnaitis, R.; Alonso, S. G.; Klüver, N.; Kühne, R.; Tyle, H.; Knecht, J. de; Dang, Z.; et al. Applicability of the Fish Embryo Acute Toxicity (FET) Test (OECD 236) in the Regulatory Context of Registration, Evaluation, Authorisation, and Restriction of Chemicals (REACH). Environ. Toxicol. Chem. 2018, 37 (3), 657-670. https://doi.org/10.1002/etc.4055.

(7) Brown, A. R.; Green, J. M.; Moreman, J.; Gunnarsson, L. M.; Mourabit, S.; Ball, J.; Winter, M. J.; Trznadel, M.; Correia, A.; Hacker, C.; et al. Cardiovascular Effects and Molecular Mechanisms of Bisphenol A and Its Metabolite MBP in Zebrafish. Environ. Sci. Technol. 2019, 53 (1), 463-474. https://doi.org/10.1021/acs.est.8b04281.

(8) Wang, P.; Xia, P.; Yang, J.; Wang, Z.; Peng, Y.; Shi, W.; Villeneuve, D. L.; Yu, H.; Zhang, X. A Reduced Transcriptome Approach to Assess Environmental Toxicants Using Zebrafish Embryo Test. Environ. Sci. Technol. 2018, 52 (2), 821-830. https://doi.org/10.1021/acs.est.7b04073.

(9) Yang, L.; Kemadjou, J. R.; Zinsmeister, C.; Bauer, M.; Legradi, J.; Müller, F.; Pankratz, M.; Jäkel, J.; Strähle, U. Transcriptional Profiling Reveals Barcode-like Toxicogenomic Responses in the Zebrafish Embryo. Genome Biol. 2007, 8 (10), R227. https://doi.org/10.1186/gb-2007-8-10-r227.

(10) Huang, S. S. Y.; Benskin, J. P.; Veldhoen, N.; Chandramouli, B.; Butler, H.; Helbing, C. C.; Cosgrove, J. R. A Multi-Omic Approach to Elucidate Low-Dose Effects of Xenobiotics in Zebrafish (Danio Rerio) Larvae. Aquat. Toxicol. 2017, 182 (Supplement C), 102-112. https://doi.org/10.1016/j.aquatox.2016.11.016.

(11) Shi, X.; Yeung, L. W. Y.; Lam, P. K. S.; Wu, R. S. S.; Zhou, B. Protein Profiles in Zebrafish (Danio Rerio) Embryos Exposed to Perfluorooctane Sulfonate. Toxicol. Sci. 2009, 110 (2), 334-340. https://doi.org/10.1093/toxsci/kfp111.

(12) Falisse, E.; Voisin, A.-S.; Silvestre, F. Impacts of Triclosan Exposure on Zebrafish Early-Life Stage: Toxicity and Acclimation Mechanisms. Aquat. Toxicol. 2017, 189, 97-107. https://doi.org/10.1016/j.aquatox.2017.06.003.

(13) Fu, J.; Gong, Z.; Kelly, B. C. Metabolomic Profiling of Zebrafish (Danio Rerio) Embryos Exposed to the Antibacterial Agent Triclosan. Environ. Toxicol. Chem. 2019, 38 (1), 240-249. https://doi.org/10.1002/etc.4292.

(14) Ramirez, T.; Daneshian, M.; Kamp, H.; Bois, F. Y.; Clench, M. R.; Coen, M.; Donley, B.; Fischer, S. M.; Ekman, D. R.; Fabian, E.; et al. Metabolomics in Toxicology and Preclinical Research. ALTEX 2013, 30 (2), 209-225.

(15) Huang, S. S. Y.; Benskin, J. P.; Chandramouli, B.; Butler, H.; Helbing, C. C.; Cosgrove, J. R. Xenobiotics Produce Distinct Metabolomic Responses in Zebrafish 
Larvae (Danio Rerio). Environ. Sci. Technol. 2016, 50 (12), 6526-6535. https://doi.org/10.1021/acs.est.6b01128.

(16) Huang, S.-M.; Xu, F.; Lam, S. H.; Gong, Z.; Ong, C. N. Metabolomics of Developing Zebrafish Embryos Using Gas Chromatography- and Liquid Chromatography-Mass Spectrometry. Mol. Biosyst. 2013, 9 (6), 1372-1380. https://doi.org/10.1039/C3MB25450J.

(17) Simmons, D. B. D.; Benskin, J. P.; Cosgrove, J. R.; Duncker, B. P.; Ekman, D. R.; Martyniuk, C. J.; Sherry, J. P. Omics for Aquatic Ecotoxicology: Control of Extraneous Variability to Enhance the Analysis of Environmental Effects. Environ. Toxicol. Chem. 2015, 34 (8), 1693-1704. https://doi.org/10.1002/etc.3002.

(18) Hayashi, S.; Yoshida, M.; Fujiwara, T.; Maegawa, S.; Fukusaki, E. Single-Embryo Metabolomics and Systematic Prediction of Developmental Stage in Zebrafish. Z. Für Naturforschung C 2014, 66 (3-4), 191-198. https://doi.org/10.1515/znc-2011-3-414.

(19) Finn, J.; Hui, M.; Li, V.; Lorenzi, V.; de la Paz, N.; Cheng, S. H.; Lai-Chan, L.; Schlenk, D. Effects of Propranolol on Heart Rate and Development in Japanese Medaka (Oryzias Latipes) and Zebrafish (Danio Rerio). Aquat. Toxicol. 2012, 122123, 214-221. https://doi.org/10.1016/j.aquatox.2012.06.013.

(20) Fraysse, B.; Mons, R.; Garric, J. Development of a Zebrafish 4-Day Embryo-Larval Bioassay to Assess Toxicity of Chemicals. Ecotoxicol. Environ. Saf. 2006, 63 (2), $253-$ 267. https://doi.org/10.1016/j.ecoenv.2004.10.015.

(21) Brunius, C.; Shi, L.; Landberg, R. Large-Scale Untargeted LC-MS Metabolomics Data Correction Using between-Batch Feature Alignment and Cluster-Based within-Batch Signal Intensity Drift Correction. Metabolomics 2016, 12 (11), 173. https://doi.org/10.1007/s11306-016-1124-4.

(22) Ribbenstedt, A.; Ziarrusta, H.; Benskin, J. P. Development, Characterization and Comparisons of Targeted and Non-Targeted Metabolomics Methods. PLOS ONE 2018, 13 (11), e0207082. https://doi.org/10.1371/journal.pone.0207082.

(23) Liebisch, G.; Lieser, B.; Rahtenberg, J.; Drobnik, W.; Schmitz, G. Erratum to "HighThroughput Quantification of Phosphatidylcholine and Sphingomyelin by Electrospray Ionization Tandem Mass Spectrometry Coupled with Isotope Corrections Algorithm" [Biochimica et Biophysics Acta, 1686 (2004) 108-117]. Biochim. Biophys. Acta BBA Mol. Cell Biol. Lipids 2005, 1734 (1), 86-89. https://doi.org/10.1016/j.bbalip.2005.01.002.

(24) Ribbenstedt, A. ExpMetFilter; Zenodo, 2019. https://doi.org/10.5281/zenodo.3191553.

(25) Broeckling, C. D.; Afsar, F. A.; Neumann, S.; Ben-Hur, A.; Prenni, J. E. RAMClust: A Novel Feature Clustering Method Enables Spectral-Matching-Based Annotation for Metabolomics Data. Anal. Chem. 2014, 86 (14), 6812-6817. https://doi.org/10.1021/ac501530d.

(26) Shi, L.; Westerhuis, J. A.; Rosén, J.; Landberg, R.; Brunius, C. Variable Selection and Validation in Multivariate Modelling. Bioinformatics. https://doi.org/10.1093/bioinformatics/bty710.

(27) Smith, C. A.; Maille, G. O.; Want, E. J.; Qin, C.; Trauger, S. A.; Brandon, T. R.; Custodio, D. E.; Abagyan, R.; Siuzdak, G. METLIN: A Metabolite Mass Spectral Database. Ther. Drug Monit. 2005, 27 (6), 747. https://doi.org/10.1097/01.ftd.0000179845.53213.39.

(28) Ruttkies, C.; Schymanski, E. L.; Wolf, S.; Hollender, J.; Neumann, S. MetFrag Relaunched: Incorporating Strategies beyond in Silico Fragmentation. $J$. Cheminformatics 2016, 8 (1), 3. https://doi.org/10.1186/s13321-016-0115-9.

(29) Dührkop, K.; Fleischauer, M.; Ludwig, M.; Aksenov, A. A.; Melnik, A. V.; Meusel, M.; Dorrestein, P. C.; Rousu, J.; Böcker, S. SIRIUS 4: A Rapid Tool for Turning 
Tandem Mass Spectra into Metabolite Structure Information. Nat. Methods 2019, 16 (4), 299. https://doi.org/10.1038/s41592-019-0344-8.

(30) Dührkop, K.; Shen, H.; Meusel, M.; Rousu, J.; Böcker, S. Searching Molecular Structure Databases with Tandem Mass Spectra Using CSI: FingerID. Proc. Natl. Acad. Sci. U. S. A. 2015, 112 (41), 12580-12585.

(31) Blaženović, I.; Kind, T.; Ji, J.; Fiehn, O. Software Tools and Approaches for Compound Identification of LC-MS/MS Data in Metabolomics. Metabolites 2018, 8 (2), 31. https://doi.org/10.3390/metabo8020031.

(32) Schollée, Jennifer. NTScreeneR; 2019.

(33) Bobeszko, M.; Dygas, A.; Nalepa, I.; Barańska, J. Different Regulation of Phospholipase D Activity in Glioma C6 Cells by Sphingosine, Propranolol, Imipramine and Phorbol Ester. Cell. Signal. 2000, 12 (6), 399-404.

(34) Pascual, F.; Carman, G. M. Phosphatidate Phosphatase, a Key Regulator of Lipid Homeostasis. Biochim. Biophys. Acta 2013, 1831 (3), 514-522. https://doi.org/10.1016/j.bbalip.2012.08.006.

(35) Meier, K. E.; Gause, K. C.; Wisehart-Johnson, A. E.; Gore, A. C. S.; Finley, E. L.; Jones, L. G.; Bradshaw, C. D.; McNair, A. F.; Ella, K. M. Effects of Propranolol on Phosphatidate Phosphohydrolase and Mitogen-Activated Protein Kinase Activities in A7r5 Vascular Smooth Muscle Cells. Cell. Signal. 1998, 10 (6), 415-426. https://doi.org/10.1016/S0898-6568(97)00140-X.

(36) Emoto, K.; Umeda, M. Membrane Lipid Control of Cytokinesis. Cell Struct. Funct. 2001, 26 (6), 659-665. https://doi.org/10.1247/csf.26.659.

(37) Orrenius, S.; Zhivotovsky, B.; Nicotera, P. Calcium: Regulation of Cell Death: The Calcium-Apoptosis Link. Nat. Rev. Mol. Cell Biol. 2003, 4 (7), 552-565. https://doi.org/10.1038/nrm1150.

(38) Bazinet, R. P.; Layé, S. Polyunsaturated Fatty Acids and Their Metabolites in Brain Function and Disease. Nat. Rev. Neurosci. 2014, 15 (12), 771-785. https://doi.org/10.1038/nrn3820.

(39) Calder, P. C. N-3 Polyunsaturated Fatty Acids, Inflammation, and Inflammatory Diseases. Am. J. Clin. Nutr. 2006, 83 (6), 1505S-1519S. https://doi.org/10.1093/ajen/83.6.1505S.

(40) Aveldaño, M. I.; Garcia, S. J. P. de; Bazán, N. G. Biosynthesis of Molecular Species of Inositol, Choline, Serine, and Ethanolamine Glycerophospholipids in the Bovine Retina. J. Lipid Res. 1983, 24 (5), 628-638.

(41) Vanderhoek, J. Y.; Feinstein, M. B. Local Anesthetics, Chlorpromazine and Propranolol Inhibit Stimulus-Activation of Phospholipase A2 in Human Platelets. Mol. Pharmacol. 1979, 16 (1), 171-180.

(42) Murthy, S. N. P.; Chung, P. H.; Lin, L.; Lomasney, J. W. Activation of Phospholipase C $\varepsilon$ by Free Fatty Acids and Cross Talk with Phospholipase D and Phospholipase A2. Biochemistry 2006, 45 (36), 10987-10997. https://doi.org/10.1021/bi060648+.

(43) Gómez-Canela, C.; Miller, T. H.; Bury, N. R.; Tauler, R.; Barron, L. P. Targeted Metabolomics of Gammarus Pulex Following Controlled Exposures to Selected Pharmaceuticals in Water. Sci. Total Environ. 2016, 562 (Supplement C), 777-788. https://doi.org/10.1016/j.scitotenv.2016.03.181.

(44) Tuross, N.; Patrick, R. L. Effects of Propranolol on Catecholamine Synthesis and Uptake in the Central Nervous System of the Rat. J. Pharmacol. Exp. Ther. 1986, 237 (3), 739-745.

(45) Davis, J. A.; Gift, J. S.; Zhao, Q. J. Introduction to Benchmark Dose Methods and U.S. EPA's Benchmark Dose Software (BMDS) Version 2.1.1. Toxicol. Appl. Pharmacol. 2011, 254 (2), 181-191. https://doi.org/10.1016/j.taap.2010.10.016. 
(46) Schymanski, E. L.; Jeon, J.; Gulde, R.; Fenner, K.; Ruff, M.; Singer, H. P.; Hollender, J. Identifying Small Molecules via High Resolution Mass Spectrometry:

Communicating Confidence. Environ. Sci. Technol. 2014, 48 (4), 2097-2098. https://doi.org/10.1021/es5002105. 